\title{
Developing a Bimolecular Affinity Purification Strategy to Isolate 26S Proteasome Holocomplexes for Complex-centric Proteomic Analysis
}

Clinton $\mathrm{Yu}^{1 \#}$, Xiaorong Wang ${ }^{1 \#}$, Wenxue $\mathrm{Li}^{2}$, Yansheng Liu ${ }^{2}$, Lan Huang ${ }^{1 *}$

${ }^{1}$ Department of Physiology \& Biophysics, University of California, Irvine, Irvine, CA 92694, USA

${ }^{2}$ Yale Cancer Biology Institute, Department of Pharmacology, Yale University, West Haven, CT

06516, USA

\# These authors contribute equally

*Correspondence should be addressed to Dr. Lan Huang (lanhuang@ uci.edu)

Medical Science I, D233

Department of Physiology \& Biophysics

University of California, Irvine

Irvine, CA 92697-4560

Phone: (949) 824-8548

Fax: (949) 824-8540 


\section{SUPPLEMENTAL METHODS}

Plasmids and Cloning

Construction of pQCXIP- $\beta 7^{\mathrm{HF}}$ plasmid: Human $\beta 7$ was PCR amplified using pcsII-pb2flag (Addgene) as the template with the following primers: forward, 5‘ATGCGGCCGCATGGAGTACCTCATCGGTATCCAAG 3‘; and reverse, 5'CCTTAATTAAGGAGCCCTGTTTGGGGAAGGAATG $3^{\circ}$. The obtained fragment was confirmed by DNA sequencing. The Human $\beta 7$ DNA fragment containing a NotI site at the $5^{\text {‘ }}$ end and a PacI site at the 3' replaced the Rpn11 fragment in pQCXIP-Rpn11-HisFlag construct to form pQCXIP-h $\beta 7$ HF construct. Construction of pQCXIH-Rpn11 ${ }^{\mathrm{TB}}$ plasmid: Rpn11 was first excised

from Rpn1 $1{ }^{\mathrm{HTBH}}$-pQCXIP using NotI and PacI and then inserted into the pQCXIH vector in order to obtain Rpn11-pQCXIH. The TB fragment of HTBH-pQCXIP was then PCR-amplified using the following primers: forward, 5'-TTAATTAACGACTACGATATACCCACAACCGC-3'; reverse,

5'-GAATTCCTAAACGCCGATCTTGATTAGACCTTG-3'. The obtained fragment was confirmed by DNA sequencing, digested by PacI and EcoR I and inserted into the same treated Rpn11-pQCXIH to get Rpn11-TB-pQCXIH. These constructs contain CMV promoters.

\section{Affinity Purification of Proteasomes}

293HF- $\beta 7 /$ Rpn11-TB cells were grown to confluence in DMEM medium containing 10\% FBS and $1 \%$ Pen/strep. Before harvesting, cells were incubated with $0.025 \%$ formaldehyde for 10 min at $37^{\circ} \mathrm{C}$ \{Wang, 2017\}. The cross-linked cells were then pelleted and washed with PBS, then lysed in native lysis buffer (50 mm Tris, $100 \mathrm{~mm} \mathrm{NaCl}, 1 \mathrm{X}$ protease inhibitor, 10\% Glycerol, 1 $\mathrm{mm}$ DTT, $5 \mathrm{~mm}$ ATP, $5 \mathrm{~mm} \mathrm{MgCl2}$ ). Following centrifugation at 13,000 rpm for $15 \mathrm{~min}$ to remove cell debris and extract supernatant, 4 different proteasome purifications were performed on the 
clarified lysates: (1) incubation with streptavidin-agarose resin then washed with TBS buffer 1, (2) incubation with anti-FLAG M2 affinity gel based on the manufacturer's protocol, washed with TBS and eluted with 3X FLAG peptide in TBS, (3) incubation with streptavidin-agarose resin, washed with TBS buffer and eluted by TEV cleavage, then further purified by binding to antiFLAG affinity gel and eluted by 3X FLAG peptide, and finally (4) incubation with anti-FLAG affinity gel, washed with TBS buffer and eluted with 3x FLAG peptide, then further purified by binding to streptavidin-agarose resin and washed with TBS buffer. All purification fractions were analyzed by western blotting using $\alpha$-Rpt6, $\alpha-\beta 7, \alpha$-Flag and streptavidin-HRP. Purified proteins were reduced/alkylated and digested as described $\{$ Wang, 2018\}. Briefly, proteins were digested in $8 \mathrm{M}$ urea buffer using LysC for $4 \mathrm{~h}$ at $37^{\circ} \mathrm{C}$, followed by trypsin digestion at $37^{\circ} \mathrm{C}$ overnight after diluting urea concentration to <1.5 M. For single-step streptavidin and dual-step FLAGstreptavidin purifications, protein reduction, alkylation and digestion were performed on-bead; for single-step FLAG and dual-step streptavidin-FLAG purifications these steps were performed on FLAG peptide-eluted proteins. The resulting peptide mixtures were then extracted and desalted prior to MS analyses. A minimum of 4 biological replicates were performed for each purification strategy.

$\underline{\text { LC MS/MS for Data-Dependent Acquisitions, Protein Identification Database Searching, and }}$

\section{$\underline{\text { Label-free Quantitation }}$}

The peptide digests were subjected to LC MS/MS analysis using an UltiMate 3000 RSLC system (Thermo Fisher Scientific) coupled in-line to an Orbitrap Fusion Lumos mass spectrometer (Thermo Fisher Scientific). Reverse-phase separation was performed on a $50 \mathrm{~cm}$ x $75 \mu \mathrm{m}$ I.D. Acclaim ${ }^{\circledR}$ PepMap RSLC column. Peptides were eluted using a gradient of $4 \%$ to $25 \%$ B over 57 min at a flow rate of $300 \mathrm{~nL} / \mathrm{min}$ (solvent $\mathrm{A}: 100 \% \mathrm{H}_{2} \mathrm{O}, 0.1 \%$ formic acid; solvent $\mathrm{B}: 100 \%$ 
acetonitrile, $0.1 \%$ formic acid). Each cycle consisted of one full Fourier transform scan mass spectrum $(375-1500 \mathrm{~m} / \mathrm{z}$, resolution of 60,000 at $\mathrm{m} / \mathrm{z} 400)$ followed by 15 data-dependent MS/MS acquired in the linear ion trap with HCD at NCE 25\%. Target ions already selected for MS/MS were dynamically excluded for $30 \mathrm{~s}$. Raw spectrometric data were converted into MGF format using the MSconvert module of ProteoWizard v.3.0.21.027 \{Chambers, 2012\}. MS/MS spectra were subjected to a developmental version of ProteinProspector (v.5.19.1) for database searching using Batch-Tag against a homo sapiens database from SwissProt (2020_12) consisting of 20,418 entries. Mass tolerances for parent ions and fragment ions were set as $\pm 20 \mathrm{ppm}$ and $0.6 \mathrm{Da}$, respectively. Trypsin was set as the enzyme with two maximum missed cleavages allowed. Two variable modifications were allowed, including protein $\mathrm{N}$-terminal acetylation, methionine oxidation, and N-terminal conversion of glutamine to pyroglutamic acid. Carbamidomethylation of cysteines was selected as a constant modification. Label-free quantitation was carried out using MaxQuant as described $\{$ Cox, 2008\}. Briefly, raw files were searched using MaxQuant (v. 1.6.0.16) against a FASTA of the complete human proteome obtained from SwissProt (version from December 2020). The first search peptide tolerance was set to $20 \mathrm{ppm}$, with main search peptide tolerance set to $4.5 \mathrm{ppm}$. For quantitation, intensities were determined as the full peak volume over the retention time profile. "Unique plus razor peptides" was selected as the degree of uniqueness required for peptides to be included in quantification. The resulting iBAQ values determined through MaxQuant were to estimate the relative subcomplex composition in purified proteasomes. The average 19S/20S ratios for each type of purification were calculated based on the normalized iBAQ abundances for $19 \mathrm{~S}$ subunits and 20S subunits from each biological replicate, then averaged across all biological replicates for each purification. 


\section{LC MS/MS for Data-Independent Acquisitions and Protein Identification \& Quantitation}

Peptide digests were subjected to LC MS/MS analysis using a Thermo EASY-nLC system (Thermo Fisher Scientific) coupled on-line to an Orbitrap Fusion Lumos mass spectrometer (Thermo Fisher Scientific). Peptides were separated using reverse-phase separation and were eluted using a gradient of $4 \%$ to $37 \% \mathrm{~B}$ over $109 \mathrm{~min}$ at a flow rate of $300 \mathrm{nl} / \mathrm{min}$ (solvent $\mathrm{A}: 100 \%$ $\mathrm{H}_{2} \mathrm{O}, 0.1 \%$ formic acid; solvent $\mathrm{B}: 80 \%$ acetonitrile, $20 \% \mathrm{H}_{2} \mathrm{O}, 0.1 \%$ formic acid) with the temperature-controlled at $60{ }^{\circ} \mathrm{C}$ using a column oven kit (PRSO-V1, Sonation GmbH, Biberach, Germany). Each cycle consisted of one full Fourier transform scan mass spectrum $(350-1650 \mathrm{~m} / \mathrm{z}$, resolution of 120,000 at $\mathrm{m} / \mathrm{z}$ 200) followed by 33-variable window schema data-independent MS/MS acquired in the Orbitrap with HCD at NCE 28 at top speed for 3s and covering the precursor mass range $350-1650 \mathrm{~m} / \mathrm{z}$. The MS/MS resolution was set to 30,000 at $\mathrm{m} / \mathrm{z} 200$ with scan range 200-1800 m/z; both MS1 and MS2 spectra were recorded in profile mode. DIA-MS data analyses were performed using Spectronaut v.14 \{Bruderer, 2015; Bruderer, 2017\} with the "DirectDIA" pipeline (i.e., an optimal spectral library-free pipeline $\{$ Tsou, 2015\}) against a SwissProt homo sapien protein database (September 2020, 20,375 entries). Methionine oxidation and protein N-terminal acetylation were set as variable modifications, whereas cysteine carbamidomethylation was set as a fixed modification. Both peptide- and protein- FDR (based on Q-value) were controlled at 1\%, and the data matrix was filtered by Q-value. The Top3 most intensive peptide precursors were used for label-free quantification at the protein level \{Wilhelm, $2014\}$. Based on the purpose of subunit abundance estimation, no run-wise normalization was performed in Spectronaut. All other Spectronaut settings for identification and quantification $\{$ Gao, 2021\} were kept as default. 


\section{$\underline{\text { Proteasome Proteolytic Activity Assays }}$}

In-solution proteolytic activity assays for purified proteasomes were performed using fluorogenic peptide substrates SUC-LLVY-AMC, SUC-LLE-AMC, and SUC-ARR-AMC, as described previously 1 . Briefly, $10 \mu \mathrm{L}$ of each sample were incubated with $100 \mu \mathrm{M}$ of substrate for $30 \mathrm{~min}$ at $37{ }^{\circ} \mathrm{C}$, followed by quenching by $1 \%$ SDS. The fluorescence of each sample was then measured at an excitation of $380 \mathrm{~nm}$ and emission of $460 \mathrm{~nm}$. The proteasome complexes in the FLAG peptide elute and bound to streptavidin agarose resin were analyzed by western blotting using streptavidin-HRP and $\alpha$-Rpt6. The proteasome activity determined through fluorogenesis was then normalized by their respective band intensities. Three biological replicates were performed, and three technical replicates were analyzed for each biological replicate. 


\section{SUPPLEMENTAL FIGURE LEGENDS}

Figure S1. Immunoblot analysis of purified proteasomes. Proteasomes obtained by all four purification strategies were analyzed by western blot using $\alpha$-Rpt6, $\alpha-\beta 7, \alpha$-Flag and streptavidinHRP. Equivalent percentages of elution were loaded in each lane. Resulting band intensities were compared between respective single- and two-step purifications (i.e. FLAG-only versus FLAGStrep, Strep-only versus Strep-FLAG). Strep-HRP bands were omitted for Strep-only/Strep-FLAG comparison as biotin is removed during TEV elution.

Figure S2. DDA-based quantitative assessment of 26S proteasome subunit abundances for each purification. All values normalized to total proteasome abundance.

Figure S3. Pair-wise correlation plots of 26S proteasome subunit abundances in all FLAG-only purifications determined by DDA $(n=6)$.

Figure S4. Pair-wise correlation plots of $26 \mathrm{~S}$ proteasome subunit abundances in all FLAG-Strep purifications determined by DDA $(\mathrm{n}=10)$.

Figure S5. Pair-wise correlation plots of $26 \mathrm{~S}$ proteasome subunit abundances in all Strep-only purifications determined by DDA $(\mathrm{n}=10)$.

Figure S6. Pair-wise correlation plots of $26 \mathrm{~S}$ proteasome subunit abundances in all Strep-FLAG purifications determined by DDA $(n=6)$.

Figure S7. DIA-based quantitative assessment of 26S proteasome subunit abundance for each purification. All values normalized to total proteasome abundance.

Figure S8. Pair-wise correlation plots of $26 \mathrm{~S}$ proteasome subunit abundances in all FLAG-only purifications determined by DIA $(n=6)$. 
Figure S9. Pair-wise correlation plots of $26 \mathrm{~S}$ proteasome subunit abundances in all FLAG-Strep purifications determined by DIA $(\mathrm{n}=10)$.

Figure S10. Pair-wise correlation plots of 26S proteasome subunit abundances in all Strep-only purifications determined by DIA $(n=6)$.

Figure S11. Pair-wise correlation plots of 26S proteasome subunit abundances in all Strep-FLAG purifications determined by DIA $(n=6)$. 


\section{SUPPLEMENTAL FIGURES}

Figure S1

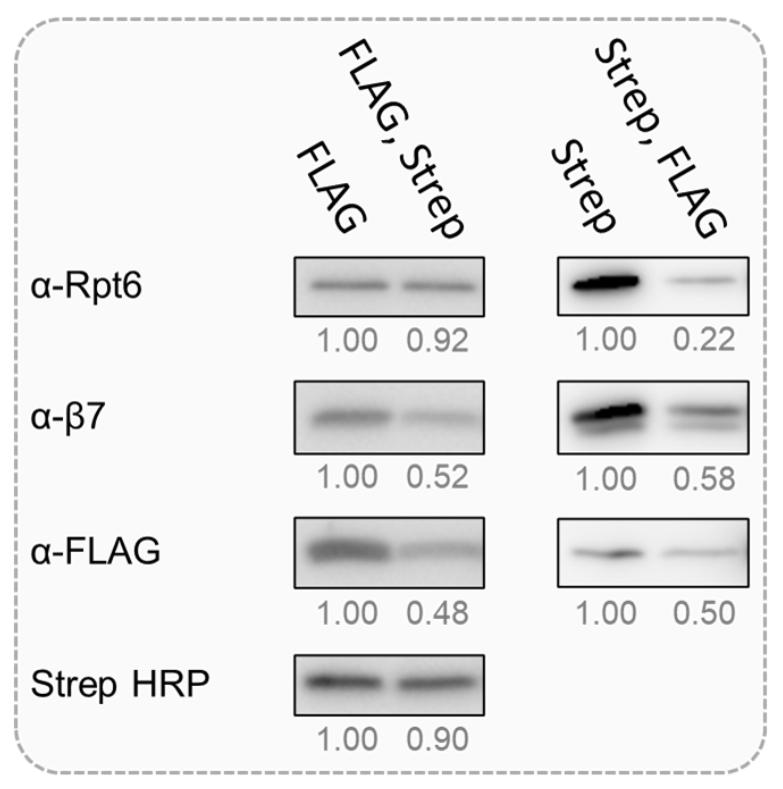




\section{Figure S2}
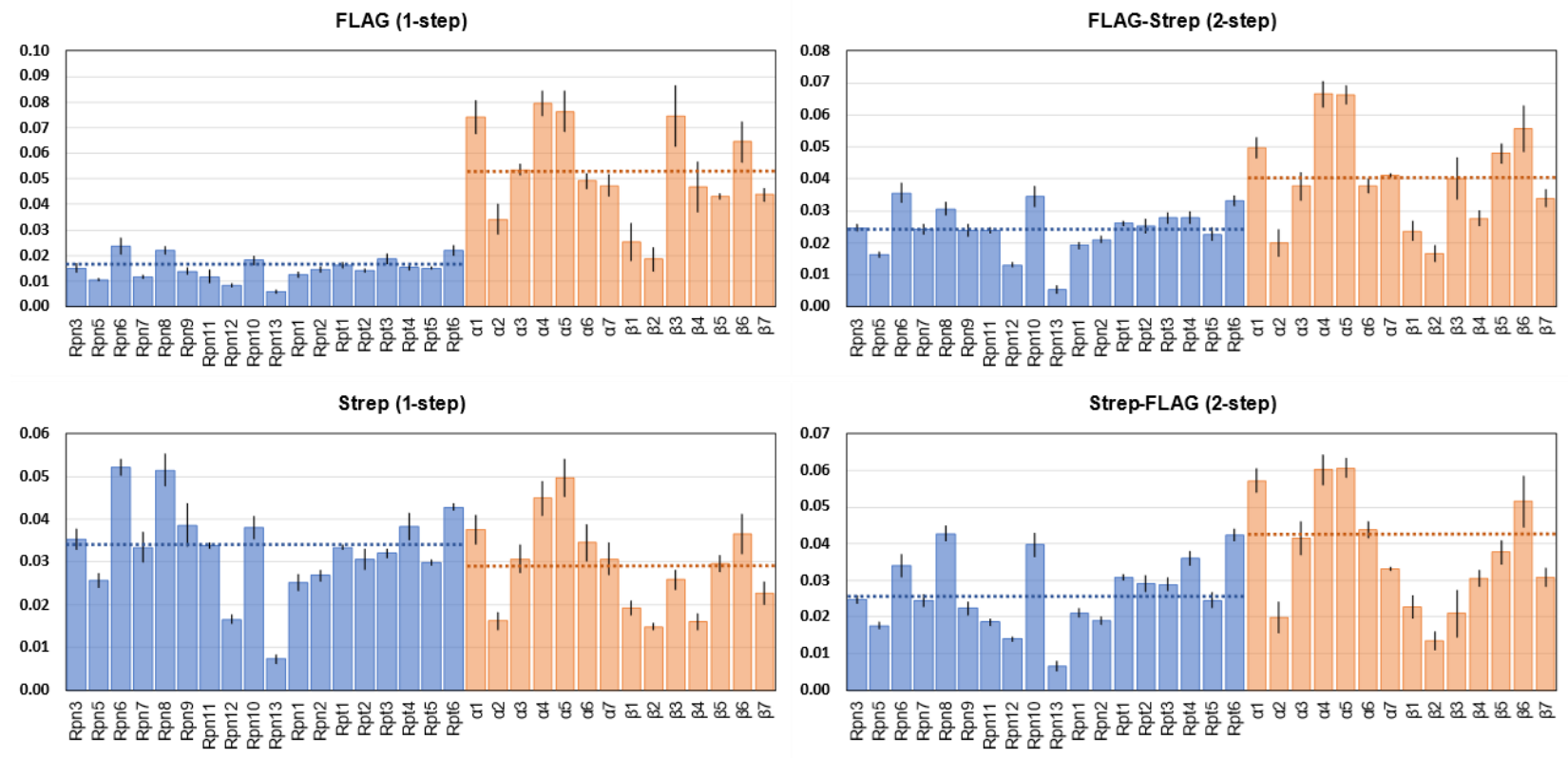
Figure S3
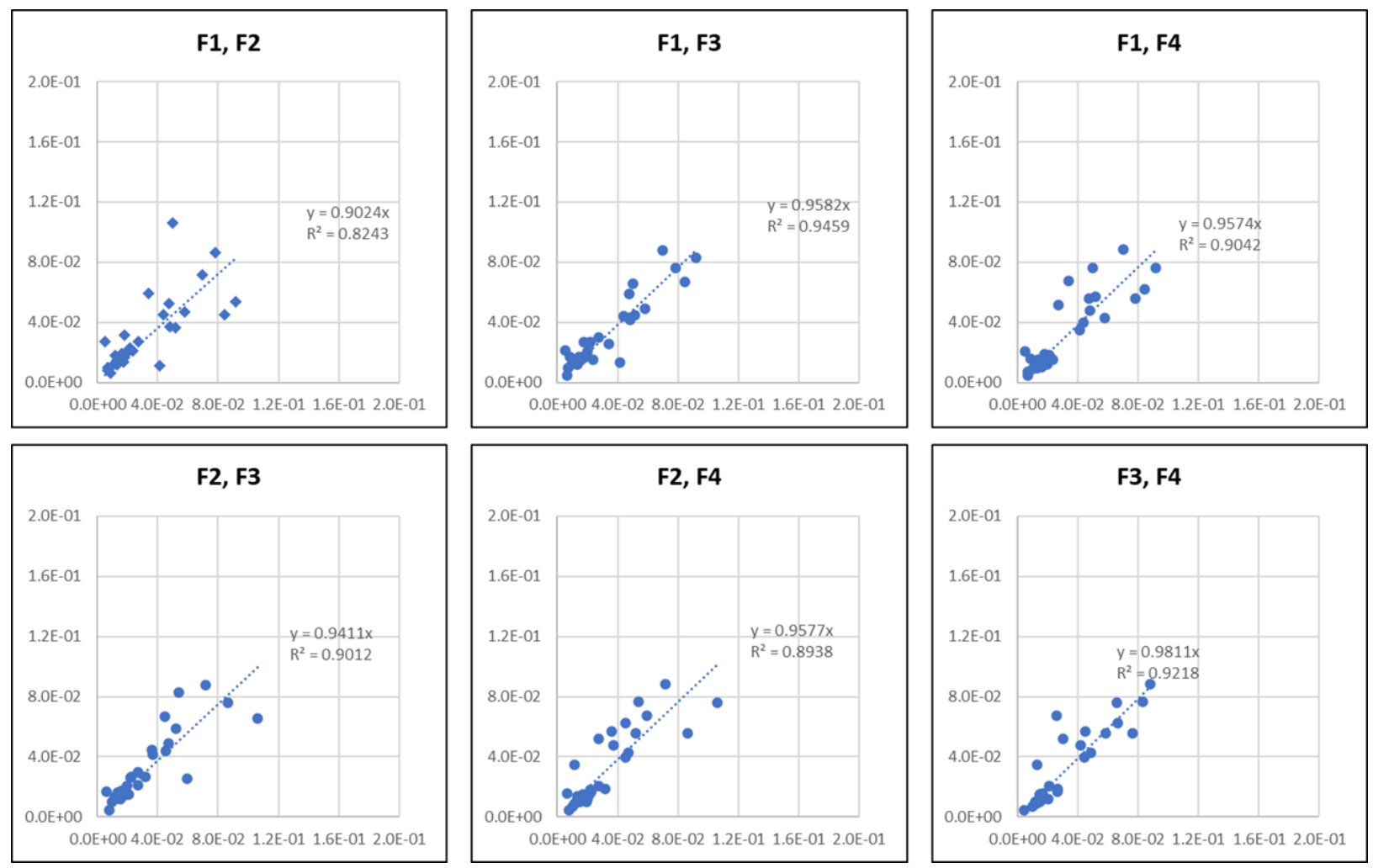
Figure S4
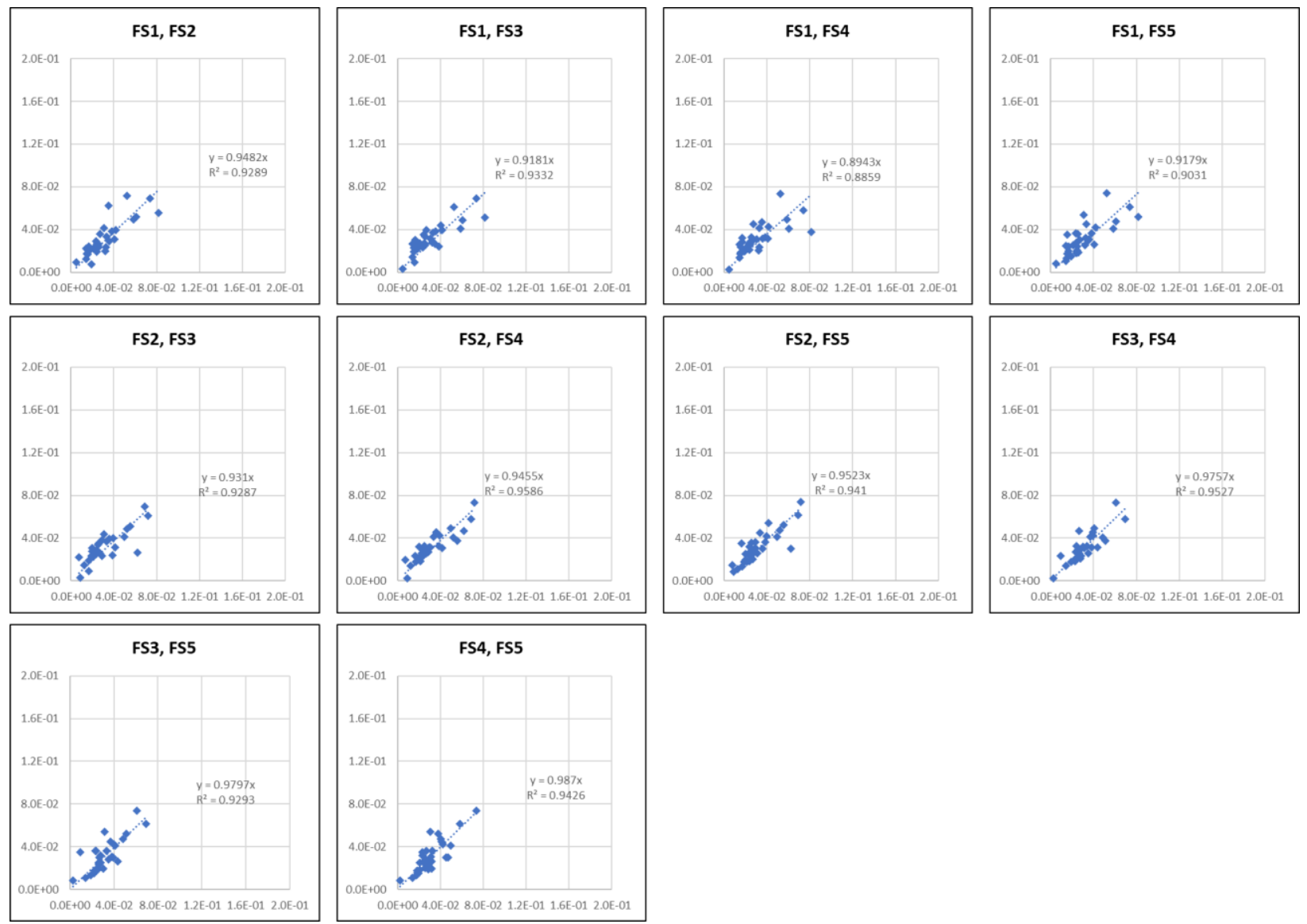
Figure S5
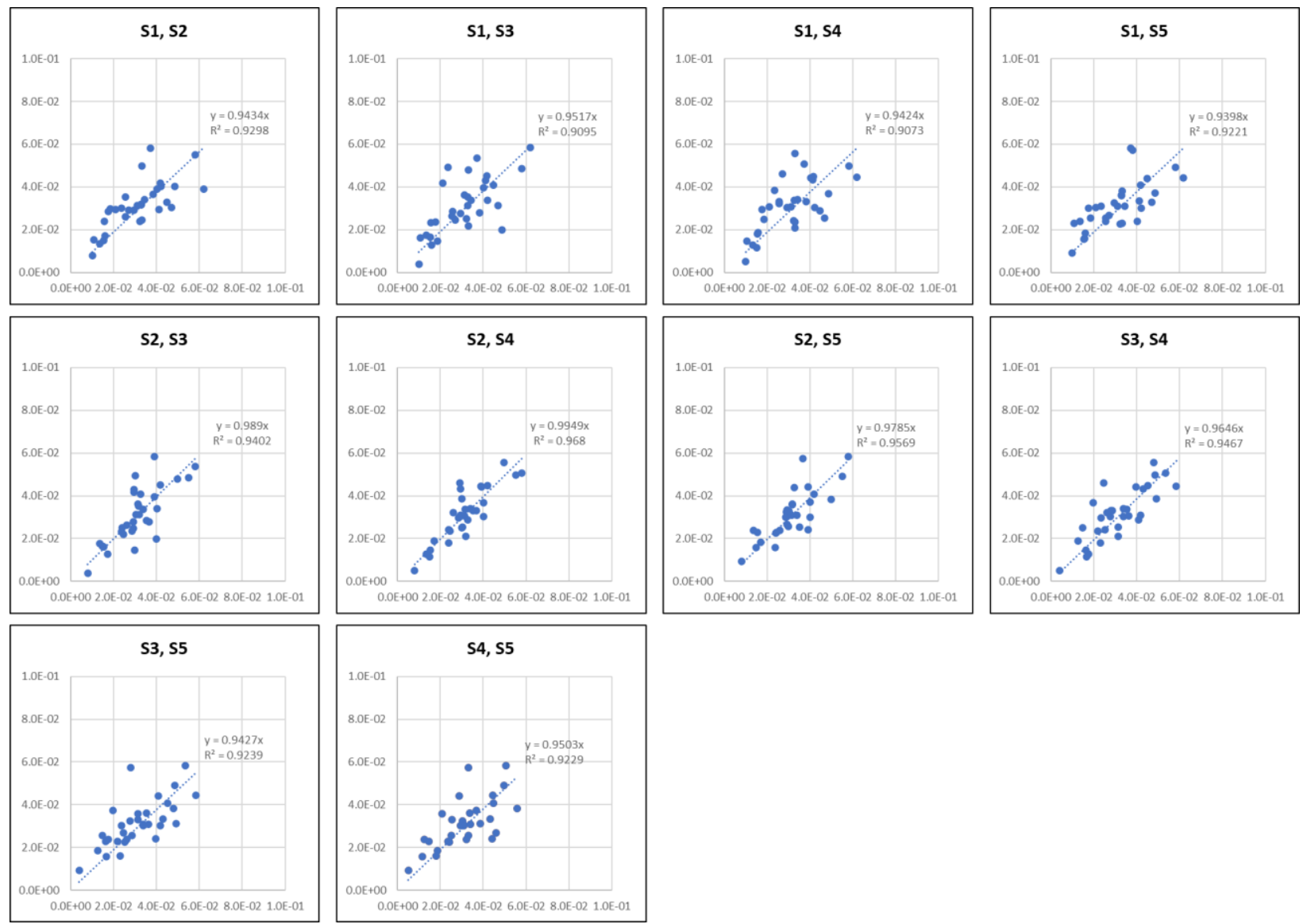
Figure S6
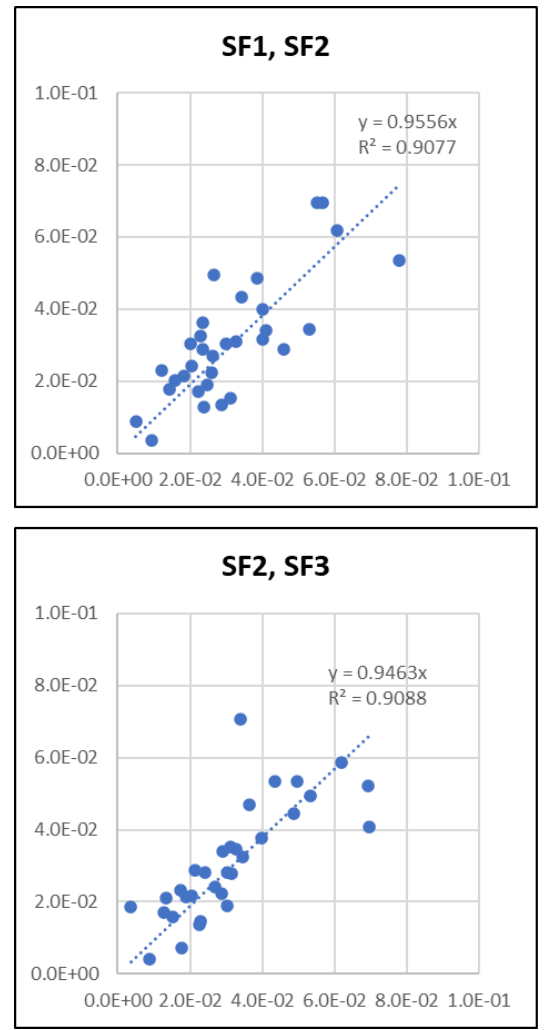
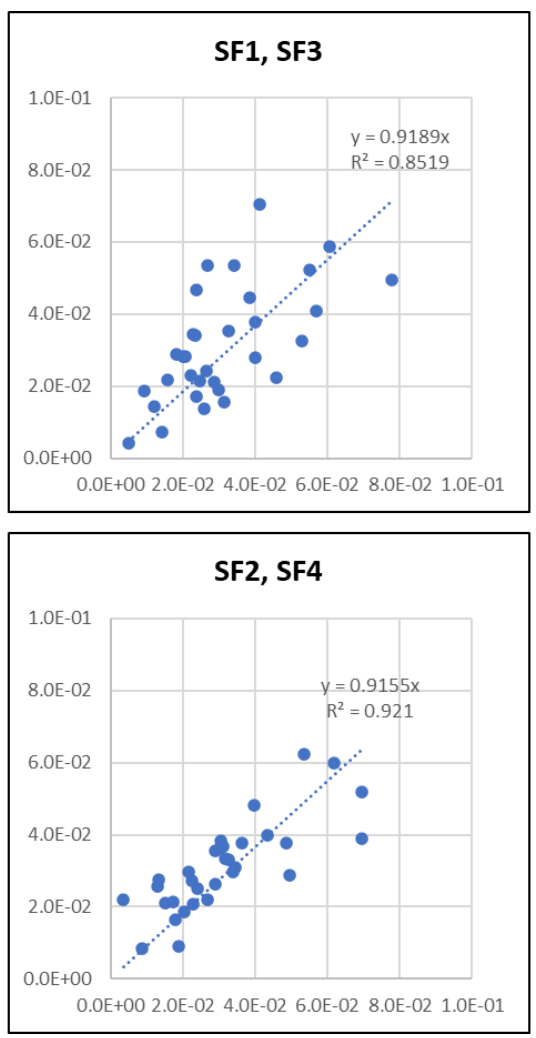
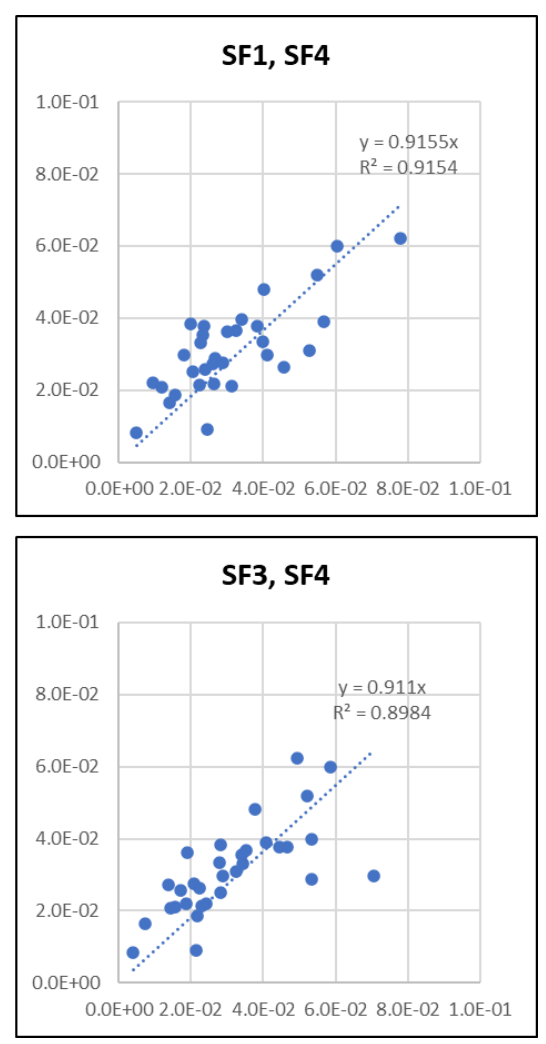


\section{Figure S7}
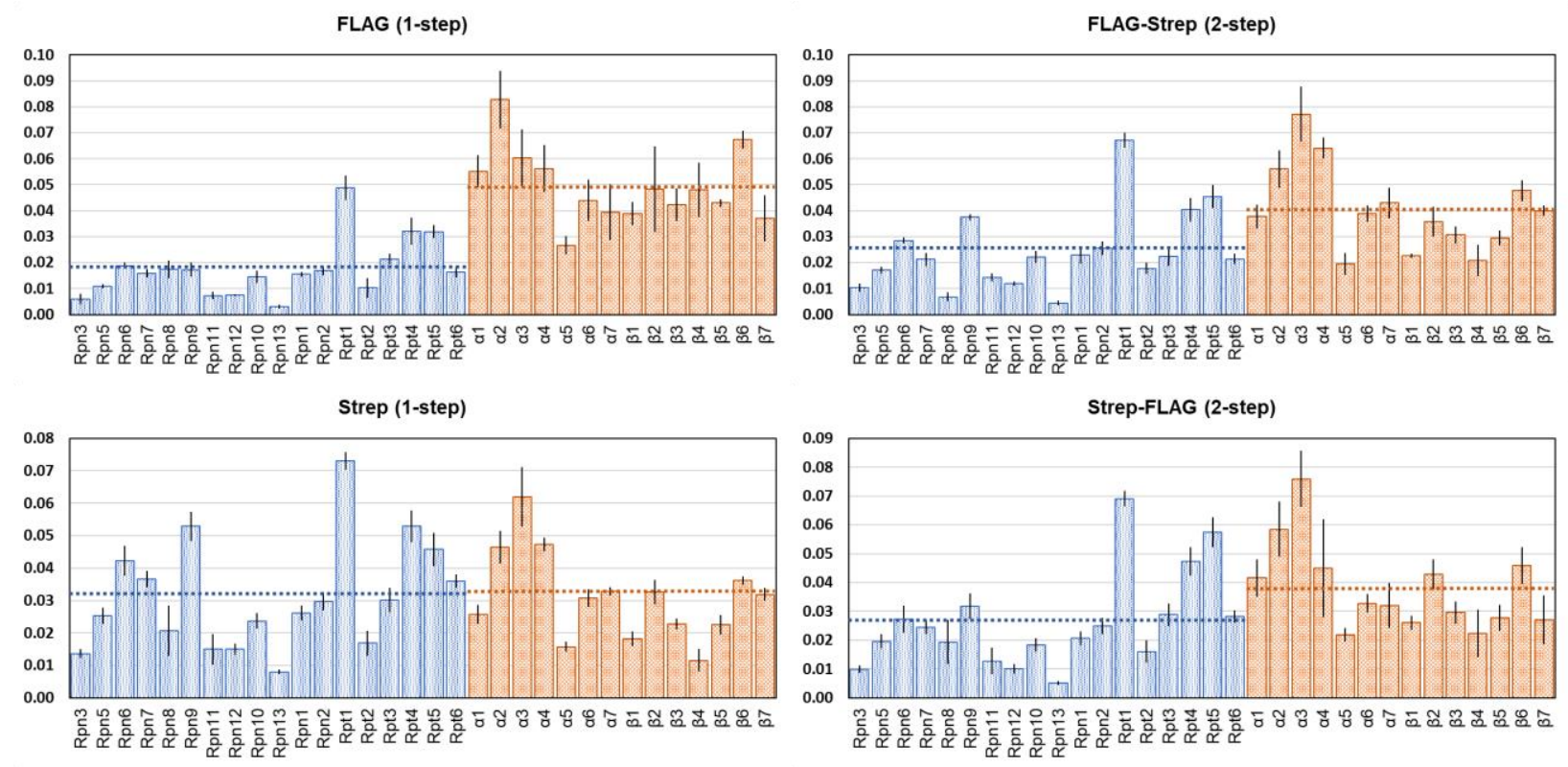
Figure S8
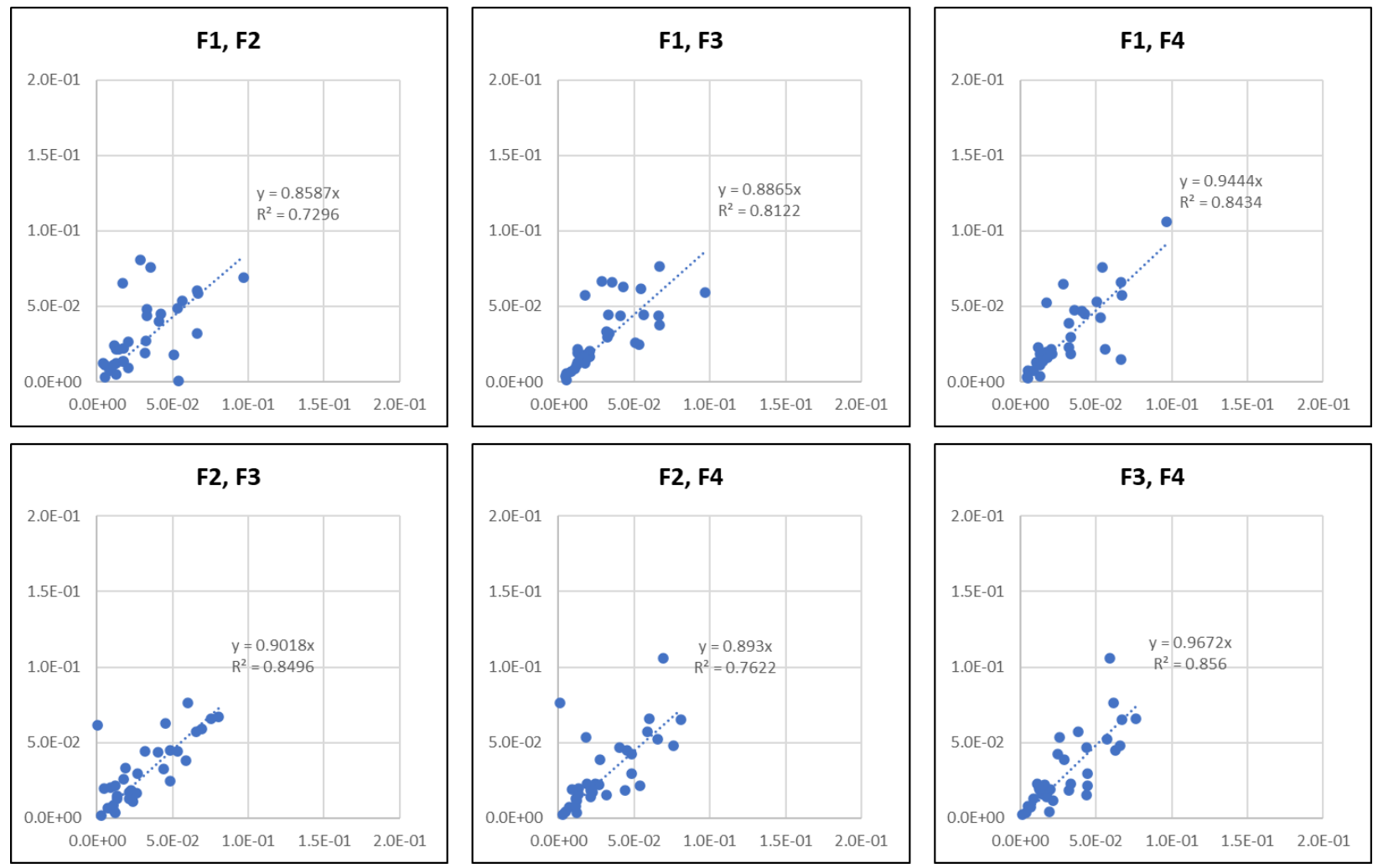

F3, F4

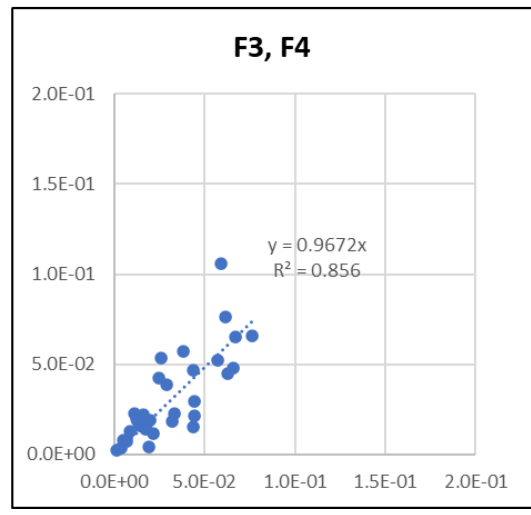


Figure S9
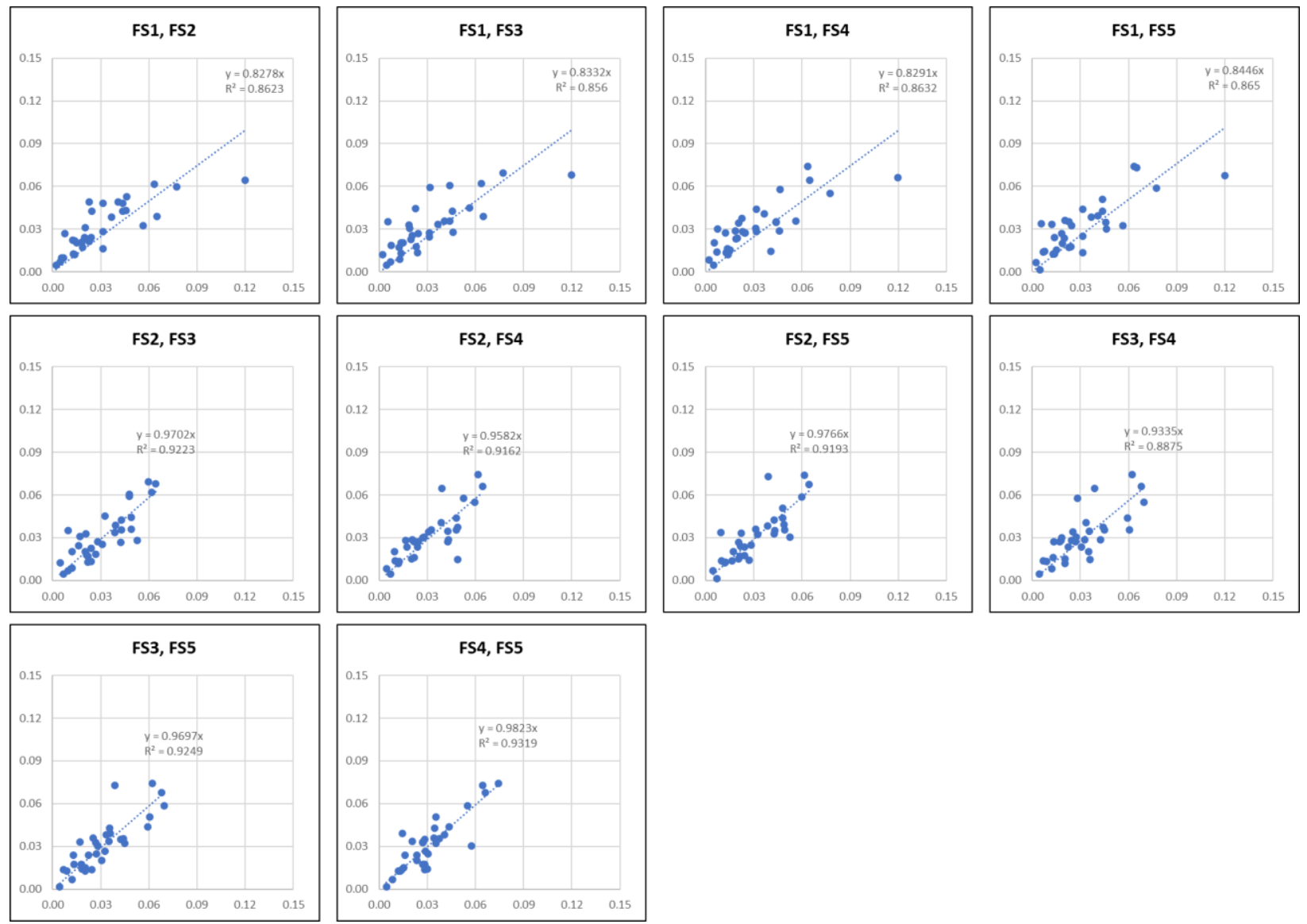
Figure S10
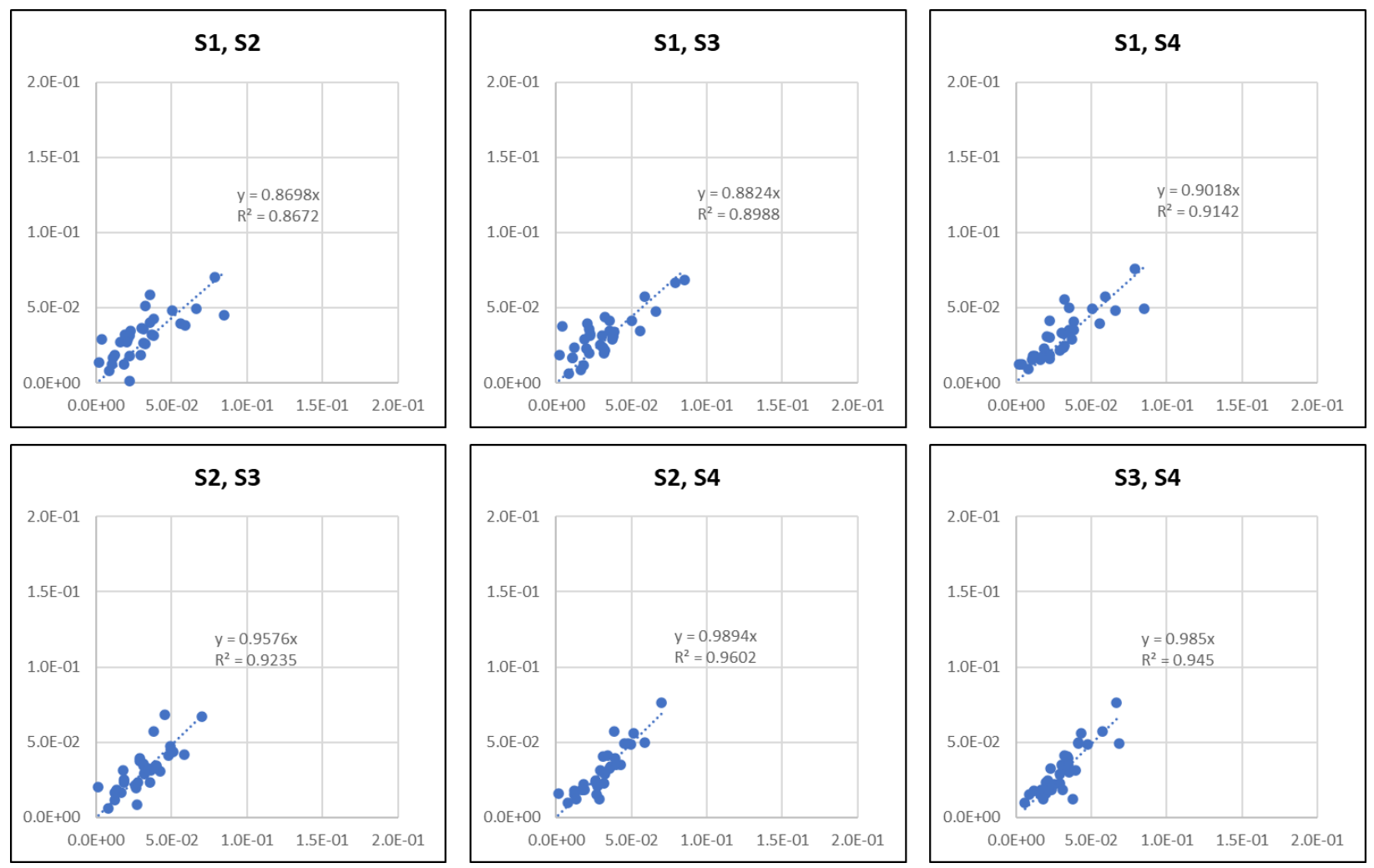


\section{Figure S11}
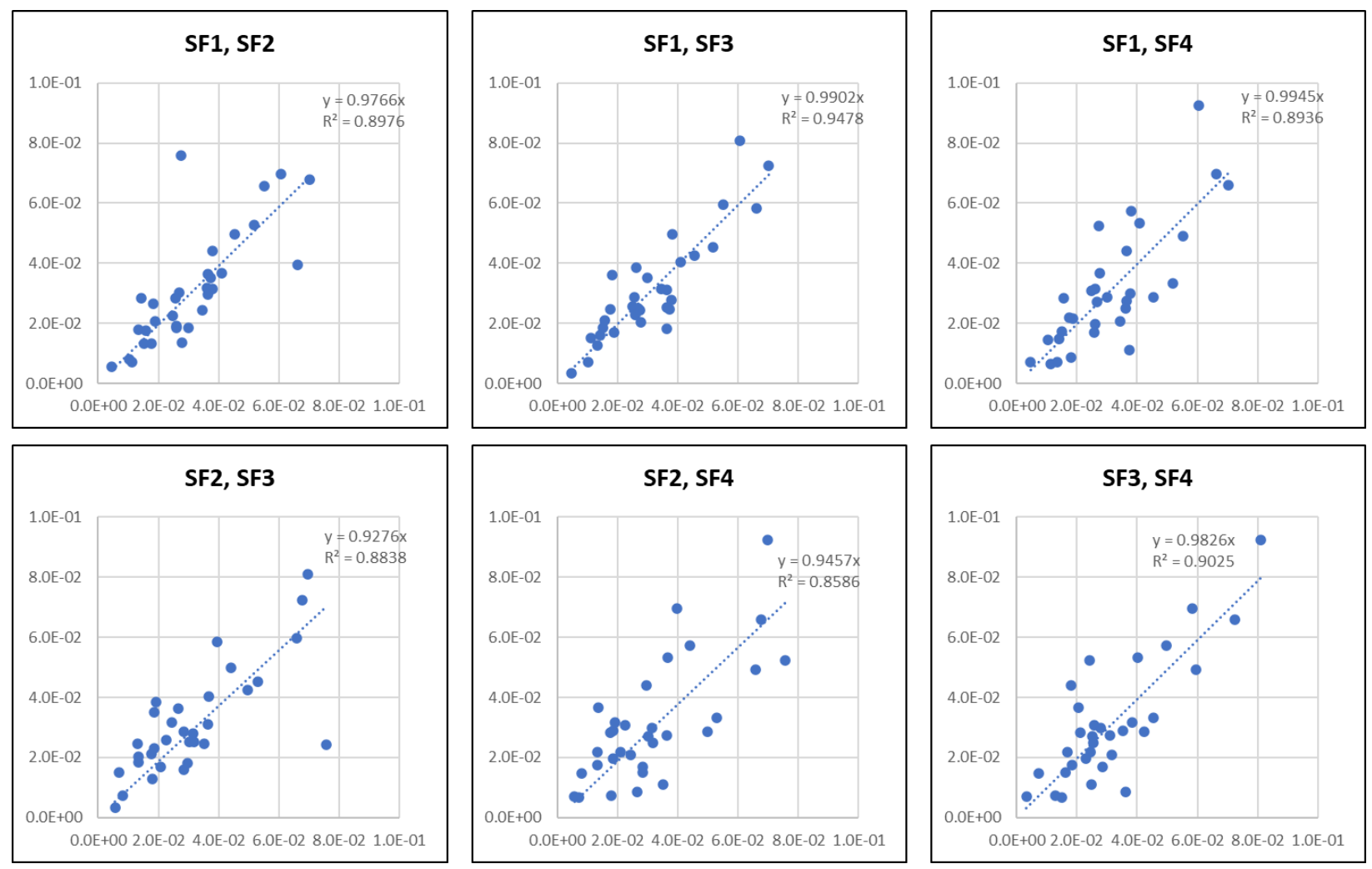